\title{
The Ising on the Tree: The Master Model for Learning, Assessment, and Navigation
}

\author{
Benjamin Deonovic, Timo Bechger, Gunter Maris
}

August 28, 2020

\begin{abstract}
Learning and assessment are intrinsically linked. However, the research, tools, and statistical models used within the two fields differ greatly. This has created a disconnect: The goals and missions of educational institutions are codified in the language and ideas of learning, but evaluated, monitored, and administered with the tools of assessment. We propose a novel statistical model, the Master model, capable of being the engine behind a modern learning and assessment system. The Master model combines three key concepts from the assessment and learning literature from the past century: A learning model should be multidimensional and hierarchical and should incorporate learning progressions. The Master model is a multidimensional latent variable model, more specifically a latent class model, that not only ranks learners from best to worst but also provides detailed diagnostic feedback to tell learners what they know, and more importantly, what they don't know. By incorporating a hierarchical structure of the latent variables, the Master model reproduces the positive manifold, a phenomenon that continues to be replicated in assessment data where scores between cognitive tests correlate positively. Finally, expert and data-driven annotation can incorporate learning progressions directly into the latent variables. With these three key concepts, the Master model can track the estimate of a learner's latent skills, track the efficacy of various educational resources such as videos, and recommend which resources the learner should next focus on in order to maximize their learning.
\end{abstract}

\section{Introduction}

Statistical models in the psychometric literature have long been developed and used for assessment. The goal of assessment is to ascertain whether the person being assessed has particular knowledge, skills, values, beliefs, or habits. An assessment provides a measure of the degree to which the evidence provided by the individual indicates that the individual has the knowledge, skills, values, beliefs, or habits being 


\begin{tabular}{c|c|c|c} 
& Correct Answer & Incorrect Answer & \\
\hline non-master & $\left(1-\pi_{j}\right) \alpha_{i}$ & $\left(1-\pi_{j}\right)\left(1-\alpha_{i}\right)$ & $\left(1-\pi_{j}\right)$ \\
master & $\pi_{j} \beta_{i}$ & $\pi_{j}\left(1-\beta_{i}\right)$ & $\pi_{j}$ \\
\hline
\end{tabular}

Table 1: A cross tabulation of the probability of answering an item correctly or incorrectly for masters and non-masters of the corresponding skill the item is tagged to. Note. Symbols are explained in the text.

assessed. This allows individuals to be ranked. An educational system, however, may benefit more from a finer-grained diagnosis of which skills individuals lack and may help to improve these skills by providing the appropriate remediation and learning resources. The need for statistical models that provide finer-grained diagnostic information from an assessment can be seen in the rise in popularity in the psychometric literature of diagnostic classification models (Rupp et al. 2010), also known as cognitive diagnostic models (CDMs).

Elsewhere in the literature there have been calls for researchers to use advances in cognitive science to refashion educational assessments (National Research Council 2001). This desire to blend models for assessment with models of learning has been brought up many times in the past (Cronbach 1957; Pellegrino et al. 1999; Embretson and Gorin 2001; Gorin 2006; Deonovic et al. 2018). In fact, more recently, von Davier et al. (2019) took this idea one step further and, echoing sentiments from elsewhere in the literature (Savi, van der Maas, et al. 2015), proposed a framework in which research in cognitive science, educational measurement, and navigation are all considered when designing an educational platform. The thesis of von Davier et al. (2019) is simple: we must help students know where they are (assessment), where they want to go (navigation), and how to get there (learning).

The statistical model developed herein, the Master model, was constructed to power a learning system that can synthesize and integrate heterogeneous evidence from a variety of sources tagged to a hierarchical skills framework, provide learners with a fine-grained diagnosis of their mastery of these skills, and provide remediation and learning resources to help learners achieve their goals within the learning system. The Master model was developed as a tool to relate evidence provided by respondents to the skills in a hierarchical framework. The Master model is a specific type of Bayes net (Almond et al. 2015) that is robust enough to be used with any hierarchical framework of skills. It can also be viewed as a CDM with a specific distributional assumption on the latent skills, specifically a hierarchical graphical model. This innovation allows for a computationally efficient procedure for estimating the posterior probability of latent skill mastery that scales well for large numbers of latent skills.

Below, we introduce the model with a motivational example in an assessment framework, provide methods for estimating parameters, provide two applications of the model to data sets, and discuss further possible extensions for the model. Notation will be introduced as needed, but see Table 2 in the Appendix for an overview of all notation in the paper. 


\section{Motivating Example}

The problem addressed in this paper is at the heart of educational measurement. To wit, a student takes a test designed to measure a number of skills and we must use the responses to make an informed assessment of the probability that a skill is mastered and provide the student with advice to improve. We illustrate this with a toy example involving one student who takes a test consisting of $m=3$ items designed to measure $r=3$ skills. Each item is tagged to one of the three skills if it provides direct evidence for mastery of that skill. The bivariate distribution of skill and item response is given in Table 1 , with $\alpha_{i}$ the probability of a correct answer to item $i$ when the skill is not mastered, $\beta_{i}$ the probability of a correct answer to item $i$ when the skill is mastered, and $\pi_{j}$ the prior probability of mastery of skill $j$. The prior probability would normally be set to equal 0.5 to express that the distribution is unbiased in the absence of evidence provided by item responses. The item responses are in a vector $\mathbf{x}$, where $x_{i}$ is the response to item $i$, which equals one if the answer was correct and zero if not.

Suppose the student answered the first item, which is tagged to Skill 2, correctly. Using Bayes' theorem, we can now determine the posterior probability of mastery:

$$
p\left(\sigma_{2}=1 \mid x_{1}=1\right)=\frac{\pi_{2} \beta_{1}}{\pi_{2} \beta_{1}+\left(1-\pi_{2}\right) \alpha_{1}}
$$

where $\sigma_{j}=1$ indicates mastery of skill $j$ and $\sigma_{j}=0$ indicates that the skill is not yet mastered. Whether or not the odds of mastery increase depends on the item:

- $\beta_{i}>\alpha_{i}$ : An correct response is expected given mastery and the odds increase.

- $\beta_{i}<\alpha_{i}$ : An incorrect response is more likely given mastery and the odds decrease.

- $\beta_{i}=\alpha_{i}$ : We have learned nothing.

The first situation is expected for a well-constructed item. The posterior log-odds of mastery is

$$
\log \frac{p\left(\sigma_{2}=1 \mid x_{1}\right)}{p\left(\sigma_{2}=0 \mid x_{1}\right)}=w_{1}+\log \frac{\pi_{2}}{1-\pi_{2}}
$$

where

$$
w_{i}=x_{i} \log \left(\frac{\beta_{i}}{\alpha_{i}}\right)+\left(1-x_{i}\right) \log \left(\frac{1-\beta_{i}}{1-\alpha_{i}}\right),
$$

This represents the weight, or direct value, of the response to item $i$ as evidence for mastery of skill $j$. Clearly, if $\beta_{i}=\alpha_{i}, w_{i}=0$, and a response to item $i$ provides no direct evidence for mastery of skill $j$.

The student now presents a response to the second item, again as evidence for mastery of Skill 2 . We assume that the student has responded independently to each item. This means that the order of responding is immaterial and the evidence accumulates additively. Specifically,

$$
\log \frac{p\left(\sigma_{2}=1 \mid \mathbf{x}\right)}{p\left(\sigma_{2}=0 \mid \mathbf{x}\right)}=\sum_{i \in \mathbf{I}_{2}} w_{i}+\log \frac{\pi_{2}}{1-\pi_{2}}
$$




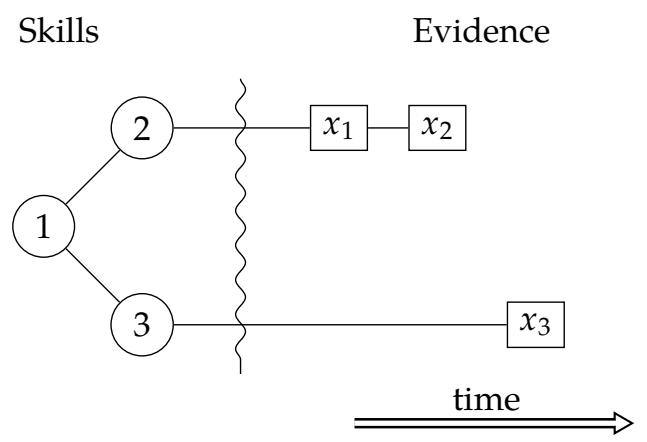

Figure 1: An illustration of the small example discussed in the introduction.

where $j_{i}$ is the index of the skill tagged to item $i$ and $\mathbf{I}_{j}=\left\{i: j_{i}=j\right\}$ denotes the set of items tagged to skill $j$. Assuming that the student has the same level of mastery when answering both questions, the evidence will set us in the right direction. The evidence is worthless if $p\left(\mathbf{x} \mid \sigma_{j}=1\right)=p\left(\mathbf{x} \mid \sigma_{j}=0\right)$ and a master and a non master are equally likely to produce the evidence.

Finally, the response to the third item is presented and here the situation changes. So far, the items provided direct evidence for mastery of the same skill. The third item, however, is tagged to another skill. If this second skill is related to the first, it raises the possibility that the third item may provide indirect or circumstantial evidence for mastery of the first skill. To make matters concrete, suppose the skills are related as in Figure 1. We see that the first and second skills, here labeled 2 and 3, are sub skills of a more abstract skill. The first two items provide evidence about the mastery of Skill 2 and the third item about Skill 3. Skill 1 is abstract in the sense that no items are directly attached to it. We will call this an untagged skill to indicate that the test provides only indirect evidence for mastery of this skill. The presence of untagged skills complicates matters further. When faced with the question of what the response pattern implies for the probability that skill 1 is mastered, intuition will clearly fail and even an evaluation of the rules of probability will rapidly become prohibitive in more realistic situations with more skills. The Master model is essentially a vehicle to evaluate the evidence in cases like this.

As we shall see in the next section, the model may be given many names. It could be classified as a Bayes net, a hierarchical latent class model, or a binary, pairwise Markov random field. We will simply call it the Master model here.

\section{The Master Model}

The Master model has two ingredients: a model for evidence given skills (the measurement model) and a model for the skills (the skill model). We first describe each model separately. For ease of notation, we assume that all test takers responded to all items and when not ambiguous we ignore the subscript for persons in $\mathrm{x}$ and $\sigma$, which correspond, respectively, to the item responses and skill masteries of an individual 
respondent. We introduce $s_{j}=2 \sigma_{j}-1$ as a $-1 /+1$ coded indicator of mastery.

The measurement model is

$$
p(\mathbf{x} \mid \sigma)=\prod_{j} \prod_{i \in \mathbf{I}_{j}}\left[\beta_{i}^{x_{i}}\left(1-\beta_{i}\right)^{1-x_{i}}\right]^{\sigma_{j}}\left[\alpha_{i}^{x_{i}}\left(1-\alpha_{i}\right)^{1-x_{i}}\right]^{1-\sigma_{j}} .
$$

The measurement model can be as complex as is needed; any CDM in the literature can be utilized. Here, we assume that every item is tagged to exactly one skill and the measurement model is a simple latent class model with multidimensional binary classes.

The Master model assumes that skills and their relationships are encoded in a hierarchical framework for skills represented by an undirected tree with real-valued connectivity matrix $\mathbf{H}$. In the motivating example, the tree was provided by the graph in Figure 1 which can be represented by the connectivity matrix

$$
\mathbf{H}=\left(\begin{array}{ccc}
0 & h_{12} & h_{13} \\
h_{21} & 0 & 0 \\
h_{31} & 0 & 0
\end{array}\right) .
$$

The entries of $\mathbf{H}$ are called edge-weights with $h_{j k} \neq 0$ if there is a connection between skill $j$ and skill $k$. The connectivity matrix is symmetric so that $h_{j k}=h_{k j}$ and its diagonal entries are zero to indicate that skill are not related to themselves. Given $\mathbf{H}$, the skill model is:

$$
p\left(\mathbf{s}_{p}\right)=\frac{1}{Z_{S}} \exp \left(\mathbf{s}_{p}^{\top} \mu+\frac{\tau}{2} \mathbf{s}_{p}^{\top} \mathbf{H} \mathbf{s}_{p}\right)
$$

where $Z_{S}=\sum_{\mathbf{s}} \exp \left(\mathbf{s}^{\top} \boldsymbol{\mu}+\frac{\tau}{2} \mathbf{s}^{\top} \mathbf{H s}\right)$ is the normalizing constant or partition function. This distribution is known as the Ising model (Lenz 1920; Ising 1925) with external magnetic field $\mu$ and inverse temperature $\tau$. The Ising model originates from statistical physics and is a popular exponential family model for binary variables that made its way into educational measurement with the recent advent of network psychometrics (van der Maas et al. 2006; Borsboom 2008; Marsman, Maris, et al. 2015; Epskamp 2017; Marsman, Borsboom, et al. 2018). While the Ising model is usually considered as a distribution of observed variables, it is used here to model the distribution of latent variables. Specifically, we use it to represent our prior beliefs and it is worth noting the distribution is unbiased in the sense that $p(\mathbf{s})=p(-\mathbf{s})$ and the marginal probability that each skill has been mastered is one half (if $\mu_{j}=0.0$ ). The relationships between the skills do, however, imply that the likelihood that skills are in the same state may be considerably higher or lower than one half. Furthermore, the probability that a skill has been mastered will change as soon as we learn whether other skills have been mastered.

Combining the measurement and skill models, we obtain the joint distribution of accuracy and skill 
mastery (note that $\sigma$ is a function of s):

$$
\begin{aligned}
p(\mathbf{s}, \mathbf{x}) & =p(\mathbf{x} \mid \sigma) p(\mathbf{s}) \\
& =\frac{1}{Z_{\mathbf{s}}} \exp \left(\log p(\mathbf{x} \mid \sigma)+\mathbf{s}^{\top} \mu+\frac{\tau}{2} \mathbf{s}^{\top} \mathbf{H s}\right) \\
& =\frac{1}{Z_{\mathbf{s}}} \exp \left(\sum_{j} e_{j}+\mathbf{s}^{\top} \mu+\frac{\tau}{2} \mathbf{s}^{\top} \mathbf{H s}\right)
\end{aligned}
$$

where $e_{j}$ is short-hand for the function

$$
\begin{aligned}
e_{j}= & \left(1-\sigma_{j}\right) \sum_{i \in \mathbf{I}_{j}}\left[x_{i} \log \alpha_{i}+\left(1-x_{i}\right) \log \left(1-\alpha_{i}\right)\right]+ \\
& \sigma_{j} \sum_{i \in \mathbf{I}_{j}}\left[x_{i} \log \beta_{i}+\left(1-x_{i}\right) \log \left(1-\beta_{i}\right)\right] \\
= & \left(1-\sigma_{j}\right) \sum_{i \in \mathbf{I}_{j}} e_{i}^{-}+\sigma_{j} \sum_{i \in \mathbf{I}_{j}} e_{i}^{+}
\end{aligned}
$$

which encodes the accumulated direct evidence with respect to skill $j$. Note that $e_{j}=0$ if none of the items are tagged to skill $j$. Note further that the interpretation of the evidence depends on whether the skill is mastered or not, and we use the terms $e_{i}^{+}$and $e_{i}^{-}$to make this explicit. It is now straightforward to derive the posterior of skill mastery given evidence:

$$
p(\mathbf{s} \mid \mathbf{x})=\frac{1}{Z_{\mathbf{x}}} \exp \left(\sum_{j} e_{j}+\mathbf{s}^{\top} \boldsymbol{\mu}+\frac{\tau}{2} \mathbf{s}^{\top} \mathbf{H s}\right)
$$

where $Z_{\mathbf{x}}=\sum_{\mathbf{s}} \exp \left(\sum_{j} e_{j}+\mathbf{s}^{\top} \boldsymbol{\mu}+\frac{\tau}{2} \mathbf{s}^{\top} \mathbf{H s}\right)$

\subsection{The Marginal Posterior Distribution for a Skill and the Strength of Indi- rect Evidence}

How does the Master model resolve the issues encountered in the motivating example? Consider the situation before we see any direct evidence related to skill $j$. The posterior is then

$$
p\left(\mathbf{s} \mid \mathbf{x}_{-j}\right)=\frac{1}{Z_{\mathbf{x}_{-j}}} \exp \left(\sum_{k \neq j} e_{k}+\mathbf{s}^{\top} \mu+\frac{\tau}{2} \mathbf{s}^{\top} \mathbf{H} \mathbf{s}\right)
$$


where $\mathbf{x}_{-j}$ is a set of responses without any items that are tagged to skill $j$ and $Z_{\mathbf{x}_{-j}}$ is the corresponding partition function. Note that

$$
\begin{aligned}
Z_{\mathbf{x}_{-j}} & =\sum_{\mathbf{s}} \exp \left(\sum_{k \neq j} e_{k}+\mathbf{s}^{\top} \boldsymbol{\mu}+\frac{\tau}{2} \mathbf{s}^{\top} \mathbf{H} \mathbf{s}\right) \\
& =\underbrace{\sum_{\mathbf{s}: s_{j}=+1} \exp \left(\sum_{k \neq j} e_{k}+\mathbf{s}^{\top} \boldsymbol{\mu}+\frac{\tau}{2} \mathbf{s}^{\top} \mathbf{H s}\right)}_{Z_{\mathbf{x}_{-j}}^{+}}+\underbrace{}_{Z_{\mathbf{x}_{-j}}^{-} \sum_{\mathbf{s} s_{j}=-1} \exp \left(\sum_{k \neq j} e_{k}+\mathbf{s}^{\top} \boldsymbol{\mu}+\frac{\tau}{2} \mathbf{s}^{\top} \mathbf{H} \mathbf{s}\right)} .
\end{aligned}
$$

It follows that

$$
\pi_{j}^{*}=p\left(s_{j}=+1 \mid \mathbf{x}_{-j}\right)=\frac{Z_{\mathbf{x}_{-j}}^{+}}{Z_{\mathbf{x}_{-j}}^{+}+Z_{\mathbf{x}_{-j}}^{-}} .
$$

This is the marginal posterior probability that skill $j$ is mastered when direct evidence with respect to this skill is not yet observed. Note the difference between $\pi_{j}$ and $\pi_{j}^{*}$. Only when the skill is untagged and unrelated to any other skill are the two probabilities equal. Otherwise, the evidence that is related to other skills affects the probability that the skill is mastered.

When direct evidence is taken into account, the marginal posterior that skill $j$ is mastered is:

$$
\begin{aligned}
p\left(s_{j}=+1 \mid \mathbf{x}\right) & =\frac{1}{Z_{\mathbf{x}}} \sum_{\mathbf{s}: s_{j}=+1} \exp \left(\sum_{k} e_{k}+\mathbf{s}^{\top} \mu+\frac{\tau}{2} \mathbf{s}^{\top} \mathbf{H} \mathbf{s}\right) \\
& =\exp \left(\sum_{i \in \mathbf{I}_{j}} e_{i}^{+}\right) \frac{Z_{\mathbf{x}_{-j}}^{+}}{Z_{\mathbf{x}}} .
\end{aligned}
$$

A similar expression can be derived for the posterior of non mastery, and it follows that:

$$
\log \frac{p\left(s_{j}=+1 \mid \mathbf{x}\right)}{p\left(s_{j}=-1 \mid \mathbf{x}\right)}=\sum_{i \in \mathbf{I}_{j}} w_{i}+\log \left(\frac{\pi_{j}^{*}}{1-\pi_{j}^{*}}\right)
$$

where $w_{i}$ is defined in Equation 3. Compare this to Equation 4. While the first term gives the value of direct evidence, the second term represents the value of the indirect evidence for mastery of skill $j$. Here we see how the Master model provides a true informative prior. If there is only one skill, $\pi_{j}^{*}=\pi_{j}=p\left(s_{j}=1\right)$, and we obtain the marginal prior that was introduced at the start of the motivating example.

\section{Estimation}

\subsection{EM Algorithm for Item Parameters}

The expectation-maximization algorithm, or EM algorithm (Dempster et al. 1977) is a special case of a minorization or MM-algorithm (Hunter and Lange 2004; Maris et al. 2014) that involves the iterative 
improvement of a surrogate function that minorizes the likelihood function. Let $\boldsymbol{\theta}=(\boldsymbol{\alpha}, \boldsymbol{\beta}, \boldsymbol{\mu}, \tau)^{\top}$, and let

$\boldsymbol{\theta}^{(t)}$ correspond to the parameter estimate at the $t$ th iteration of the EM algorithm. If each individual, $p$, is an independent realization of the model, the minorizing function is

$$
\begin{aligned}
Q\left(\boldsymbol{\theta}, \boldsymbol{\theta}^{(t)}\right) & =E_{S \mid \mathbf{x}, \boldsymbol{\theta}^{(t)}}[\log p(\mathbf{x}, \mathbf{s}) \mid \boldsymbol{\theta}] \\
& =\sum_{p=1}^{n} \sum_{j} E_{S \mid \mathbf{x}, \boldsymbol{\theta}^{(t)}}\left[e_{p j}\right]+E_{S \mid \mathbf{x}, \boldsymbol{\theta}^{(t)}}\left[\boldsymbol{\mu}^{\top} \mathbf{s}_{p}\right]+\frac{\tau}{2} E_{S \mid \mathbf{x}, \boldsymbol{\theta}^{(t)}}\left[\mathbf{s}_{p}^{\top} \mathbf{H} \mathbf{s}_{p}\right]-\log Z_{S}
\end{aligned}
$$

where

$$
E_{S \mid \mathbf{x}, \boldsymbol{\theta}^{(t)}}\left[e_{p j}\right]=\left(1-E_{S \mid \mathbf{x}, \boldsymbol{\theta}^{(t)}}\left[\sigma_{p j}\right]\right) \sum_{i \in \mathbf{I}_{j}} e_{p i}^{-}+E_{S \mid \mathbf{x}, \boldsymbol{\theta}^{(t)}}\left[\sigma_{p j}\right] \sum_{i \in \mathbf{I}_{j}} e_{p i}^{+}
$$

In this section, we consider how parameter values are chosen that optimize or at least improve the value of $Q$ - the latter giving a generalized EM algorithm. Next, we consider how the expected values are determined. Detailed derivations can be found in the Appendix.

\subsubsection{Estimating $\alpha$ and $\beta$}

For the item parameters, we obtain the following closed-form solutions at each iteration of the EM:

$$
\alpha_{i}=\frac{\sum_{p=1}^{n}\left(1-\tilde{\sigma}_{p j_{i}}\right) x_{p i}}{\sum_{p=1}^{n}\left(1-\tilde{\sigma}_{p j_{i}}\right)}
$$

where $\tilde{\boldsymbol{\sigma}}=\left(\tilde{\sigma}_{p 1}, \ldots, \tilde{\sigma}_{p r}\right)^{\top}$ and $\tilde{\sigma}_{p j}$ corresponds to the $j$ th entry of $E_{S \mid \mathbf{x}, \boldsymbol{\theta}^{(t)}}\left[\sigma_{p}\right]$. So we see that the EM estimate of the $\alpha_{i}$ is the ratio of the expected number of people who didn't master the skill associated with item $i$ but answered the item correctly divided by the expected total number of people who didn't master this skill. Similarly, for $\beta_{i}$ we get the estimator

$$
\beta_{i}=\frac{\sum_{p=1}^{n} \tilde{\sigma}_{p j_{i}} x_{p i}}{\sum_{p=1}^{n} \tilde{\sigma}_{p j_{i}}}
$$

which is the expected total number of people who have mastered the skill associated with item $i$ and got item $i$ correct divided by the total number of people expected to have mastered the skill.

\subsubsection{Inverse temperature $\tau$ and external magnetic field $\mu$}

Estimation is slightly more involved for the temperature and the magnetic field. Both involve running a Newton-Raphson optimization at each iteration of the EM algorithm. If we perform only one step of the 
Newton-Raphson optimization, we obtain the following closed form solutions:

$$
\begin{aligned}
& \tau=\frac{1}{n} \sum_{p=1}^{n} E_{S \mid \mathbf{x}, \boldsymbol{\theta}^{(t)}}\left[\frac{1}{2} \mathbf{s}_{p}^{\top} \mathbf{H} \mathbf{s}_{p}\right] /\left(\frac{1}{2} \sum_{j_{1} \neq j_{2}} h_{j_{1} j_{2}}^{2}\right) \\
& \boldsymbol{\mu}=\frac{1}{n} \sum_{p=1}^{n} E_{S \mid \mathbf{x}, \boldsymbol{\theta}^{(t)}}\left[\mathbf{s}_{p}\right] .
\end{aligned}
$$

Under the standard regularity conditions, only performing one step of the Newton-Raphson algorithm is not guaranteed to maximize $Q$ at each iteration of the EM algorithm, but is expected to only increase $Q$, resulting in a generalized EM algorithm.

\subsubsection{Estimating the connectivity matrix $H$}

The connectivity matrix encodes the design of the assessment, and so far we have assumed that the values of $\mathbf{H}$ were known. We can relax this assumption. Suppose that the indices for which $h_{j k}=0$ were known, but we want to freely estimate $h_{j k} \neq 0$. If $\mathbf{H}$ is a tree structure and $\boldsymbol{\mu}=\mathbf{0}$, then using a result from Griffiths (1967), we show that the closed-form solution for the edge weight is

$$
h_{j k}=\frac{1}{\tau} \operatorname{atanh}\left(\frac{1}{n} \sum_{p=1}^{n} E_{S \mid \mathbf{x}, \boldsymbol{\theta}^{(t)}}\left[s_{p j} s_{p k}\right]\right)
$$

which shows that the edge weights reflect the correlation between the corresponding skills.

It should be noted in this instance that the values of $\mathbf{H}$ and the inverse temperature parameter $\tau$ are not simultaneously identified by the observations. Thus, if one wishes to estimate the values for which $h_{j k} \neq 0$ freely, one would have to hold $\tau$ constant.

\subsection{Belief Propagation}

The normalizing constant $Z_{S}$ is computationally intractable as it involves the sum over $2^{k}$ different configurations of skill mastery. It is difficult, therefore, to compute the expected values and the conditional expected values that are required in the EM algorithm. One way around this is to sample from the model and compute an empirical estimate of the expectation. This is typically referred to as Monte Carlo EM (Wei and Tanner 1990). The number of draws can be a single draw from the model, in which case the approach is referred to as stochastic EM (Celeux and Diebolt 1992). Such an approach can be computationally challenging. Here, we discuss an approach called belief propagation capable of providing exact conditional expectations in a computational efficient and scalable manner.

Belief propagation (BP) is a popular algorithm for approximate inference (in general graphs), or exact inference in tree graphs. An undirected graph, $G=\{E, V\}$, is composed of a set of vertices, $V$, and edges, $E$. Each edge in the graph describes a bidirectional relationship between two vertices in the graph. The set of 
latent skills in the Master model can be considered a graph where the vertices correspond to the skills in the Master model and an edge exists between skills $j$ and $k$ if $h_{j k} \neq 0$. A tree graph is a graph where there exists a unique set of edges to get from one vertex another for every pair of vertices in the graph, (i.e., there are no loops in the graph). In a directed graph, the relationship between two vertices (the edge) is unidirectional. One of the vertices is called the parent and the other the child. In a directed tree graph, there exists a root vertex, which does not have any parent nodes, and leaf vertices, which do not have any child nodes.

To proceed, we will assume that $\mathbf{H}$ is a directed tree graph. Note that we can factor the posterior of skill mastery given evidence in Equation 13 in the following way:

$$
p\left(\mathbf{s}_{p} \mid \mathbf{x}_{p}\right)=\frac{1}{Z_{\mathbf{x}}}(\prod_{j} \underbrace{\exp \left(e_{p j}+\mu_{j} s_{j}\right)}_{\phi_{j}\left(s_{p j}\right)})(\prod_{j_{1}<j_{2}} \underbrace{\exp \left(\tau h_{j_{1} j_{2}} s_{p j_{1}} s_{p j_{2}}\right)}_{\phi_{j_{1} j_{2}}\left(s_{j_{1}}, s_{j_{2}}\right)})
$$

where $\phi_{j}(s)$ is called the unary potential and $\phi_{j_{1} j_{2}}(s, u)$ is called the edge or pairwise potential. In $\mathrm{BP}$, messages are passed between variables. With a tree-structured undirected graph, passing messages from the leaves of the tree to the root, and then from the root back to the leaves will result in calculating the exact marginal distributions, $P\left(S_{j} \mid \mathbf{x}_{p}\right)$, at each node. The message from node $j_{1}$ to node $j_{2}$ is given by

$$
\omega_{j_{1} j_{2}}(s) \propto \sum_{u} \phi_{j_{1}}(u) \phi_{j_{1} j_{2}}(u, s) \prod_{j_{3} \in N\left(j_{1}\right) \backslash j_{2}} \omega_{j_{3} j_{1}}(u)
$$

where $N\left(j_{1}\right)$ represents the neighborhood of node $j_{1}$; that is, $\left\{j_{2}: h_{j_{1} j_{2}} \neq 0\right\}$, and the backslash denotes set difference. Since this is a marginal distribution, it should be normalized so that $\omega_{j_{1} j_{2}}(+1)+\omega_{j_{1} j_{2}}(-1)=1$. Once a node's incoming messages have been computed, the marginal distribution, called the belief in BP, can be computed with

$$
p\left(s_{j_{1}}=s \mid \mathbf{x}\right)=b_{j_{1}}(s) \propto \phi_{j_{1}}(s) \prod_{j_{2} \in N\left(j_{1}\right)} \omega_{j_{2} j_{1}}(s)
$$

Along with the expected values, we will need the pairwise expectations $E\left[S_{j} S_{j^{\prime}} \mid X\right]$. We can compute those in a similar manner using the equation

$$
b_{j_{1} j_{2}}(s, u) \propto \phi_{j_{1} j_{2}}(s, u) \phi_{j_{1}}(s) \phi_{j_{2}}(u) \prod_{j_{3} \in N\left(j_{1}\right) \backslash j_{2}} \omega_{j_{3} j_{1}}(s) \prod_{j_{3} \in N\left(j_{2}\right) \backslash j_{1}} \omega_{j_{3} j_{2}}(u) .
$$

Note that BP has been reinvented many times and in different fields. It is also known as loopy belief propagation, the sum-product algorithm, and the Bethe-Peierls approximation. One of the earliest contexts for which it was proposed was for error-correcting codes by Gallager (1963) (but also see McEliece et al. (1998)). Nakanishi (1981) describes BP for the special case of a binary, pairwise Markov random field, which is equivalent to our formulation of the Master model. In artificial intelligence, it is commonly attributed to Pearl (1988), who used it for estimating Bayes nets. For more details on belief propagation and the derivation 
of the above formulas see Almond et al. (2015).

\section{Real Data Analysis}

The key ingredient that differentiates the Master model from other latent class models that have been applied to learning systems is the hierarchical categorization of the skills involved. From over a century of large-scale assessment, we have learned that assessments have a hierarchical structure, that is, scores between cognitive tests correlate positively with one another. This was referred to as the positive manifold by Spearman (1904) and has been explored in a variety of modern literature (Undheim and Gustafsson 1987; Carroll 1993; Savi, Marsman, et al. 2019). Mathematics items correlate more with other mathematics items than with reading items. Within mathematics, algebra items correlate more with other algebra items than with geometry items, etc. Such a hierarchy is encoded into educational standards (e.g., Common Core Standards) and frameworks around which assessments are designed. A recent example of such a framework is the Holistic Model of Education and Workplace Success, also known as the Holistic Framework (HF) by Camara et al. (2015).

The HF was developed to combat the tendency of policymakers and accountability systems to focus only on academic measures when discussing college and career readiness. Research has shown that performance in college and in the workplace correlates not only with measures of traditional academic skills but also with newer measures of social and emotional learning capacity and other behavioral skills such as self-control (Hannon 2014; Stewart 2015). Camara et al. (2015) identified the need for a framework that organizes the knowledge and skills necessary to succeed in the modern world. The framework is organized into four broad domains: core academic skills, cross-cutting capabilities, behavioral skills, and education-, and career-navigation skills.

\subsection{ACT Test}

We first look at an application of the Master model to assessment data. We fit the Master model to a data set that includes 48,187 unique test takers who took the ACT®test. These test attempts span 15 unique ACT forms. Each form comprises 215 items with 75, 60, 40, and 40 pertaining to the core academic skills of English, mathematics, reading, and science, respectively. Test takers receive their raw scores on these test sections along with a scaled score with values between 1 and 36 . When fitting the Master model to this data set, we set $\tau$ to 1.0 and all the edge weights to 0.5 , and we estimate all other parameters as outlined above. Figure 2 shows a small excerpt from the HF with skills from the science skill to which one particular form had items tagged. The numbers in the nodes indicate how many items were tagged to this skill or any of its child skills. The color of the nodes represents the posterior mastery probability of one particular test taker who received a 23 scale score on the science section. The labels on the nodes represent the 


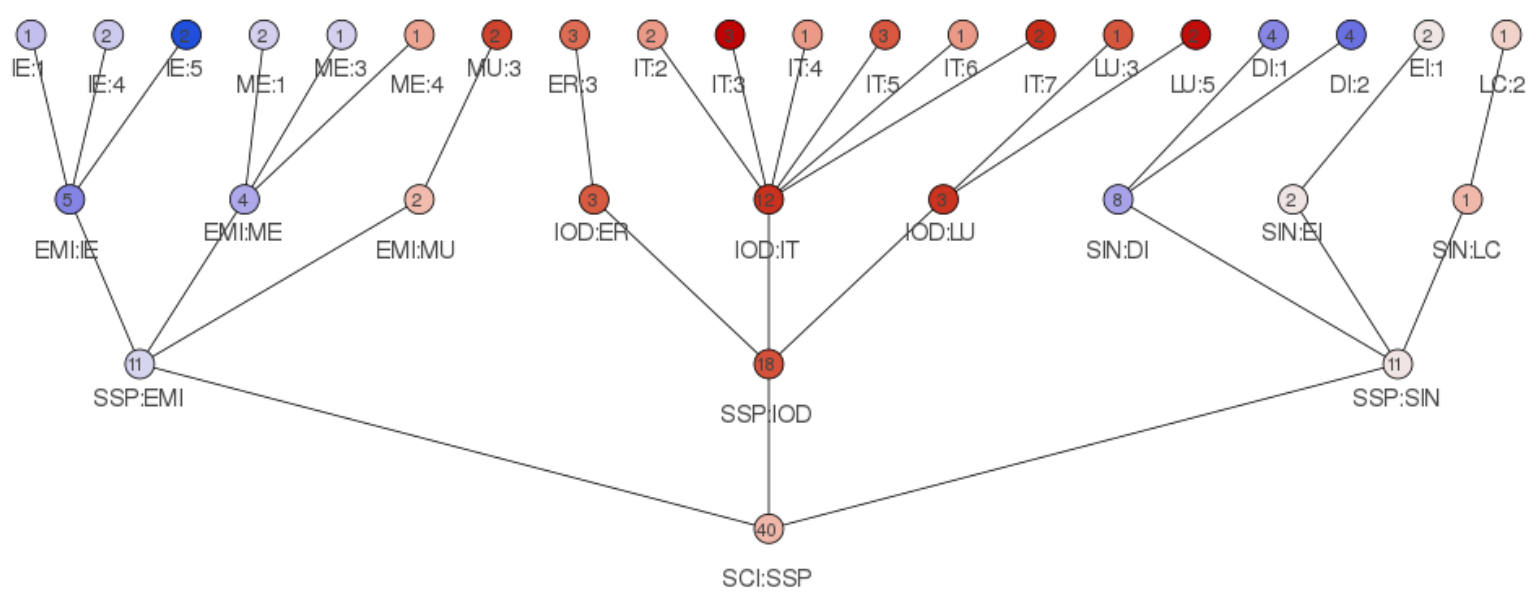

Figure 2: The nodes in the core academic skill of science to which one specific ACT science test has items tagged. Colors indicate the posterior probability of mastery (blue is closer to 1 , red is closer to 0 ) of a particular test taker. Numbers indicate how many items are tagged to this skill or one of its child skills.

names of the skills. These names can be looked up for these science skills (and all other HF skills) here: https://frameworks. act.org/cfdoc/.

Figure 2 illustrates how the Master model can be used to provide diagnostic feedback in assessments. The specific test taker represented here may not be satisfied with their performance, but simply knowing their raw score or scale score may not be sufficient to help them to know which aspects of the science test to study. Figure 2 provides the test taker with a finer-grained diagnostic. In particular, they can instantly see that the topic they struggled the most with is SSP: IOD, the interpretation of data. Once the test taker feels they have obtained sufficient remediation in this area, what should they focus on next? Overall, the model shows that they have a strong grasp of SSP:EMI, or evaluation of models, inferences, and experimental results, but incorrectly answered several items related to EMI:MU, or understanding and comparing models.

Note that Figure 2 shows only those skills that are tagged by one specific test, which is a small subset of the HF which comprises over 4,000 nodes. The Master model can estimate the mastery of the skills that did not have any items tagged to them, relying on the structure of the HF (the structure of the $\mathbf{H}$ matrix in the model) and the edge weights between skills (i.e., the values of $h_{j k}$ in the matrix), which correspond to the correlations between skills. However, estimating these edge weights requires considerable data for each pair of skills. Thus, in this small example, we did not estimate these edge weights but only focused on the skills directly tagged by the test.

Figure 3 shows the distribution of the posterior probability of mastery of the root science node broken down by the scale score achieved on the science test. This shows that there is a strong correlation (Pearson correlation between the ACT science scale score and the Master model expected mastery of science is 0.93 ) between the posterior probability of mastering science and the scale score the test taker obtained. The figure 


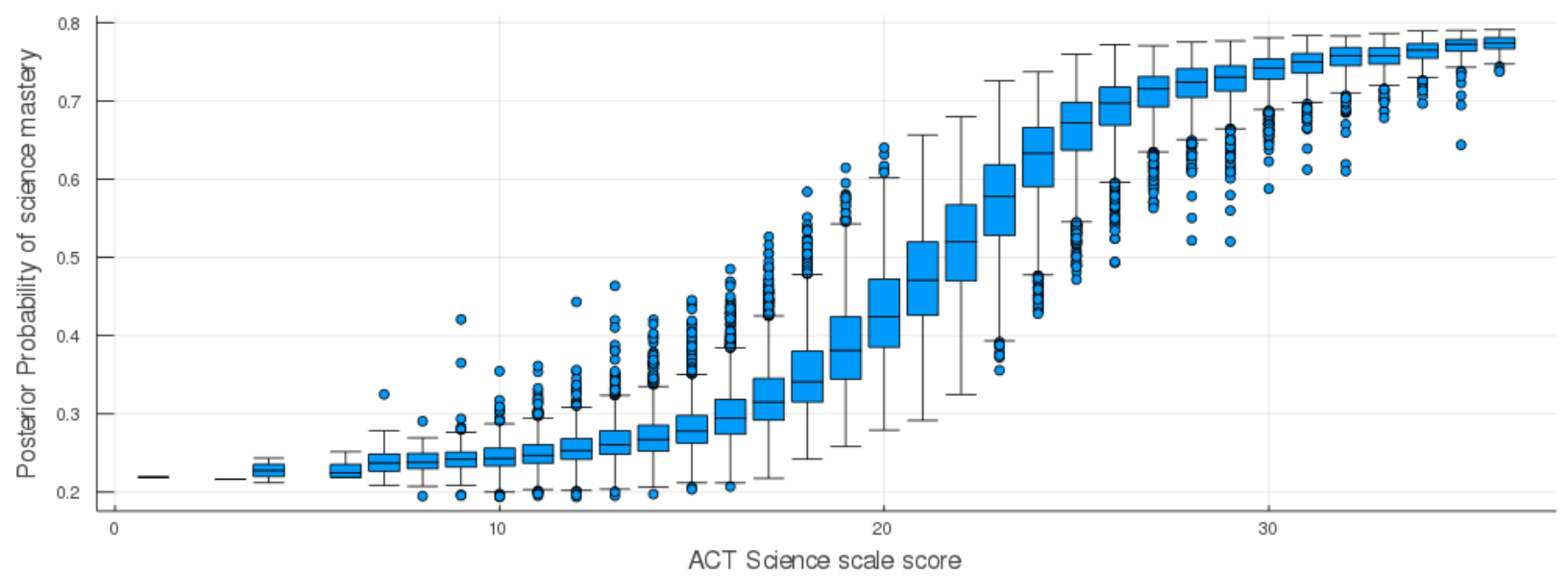

Figure 3: Distribution of posterior probability of science mastery grouped by the scale score achieved on the ACT science test.

also shows significant variability. This is because the science test has only 40 items, but the core academic skill of science has 1,546 skill nodes. Less variable estimates will be obtained once a person takes multiple assessments. However, assessments are designed to not change significantly year over year, and only cover a specific predetermined set of domains. Also, evidence provided by a person at one point in time may differ from evidence provided later if the underlying latent skills have changed. Thus, multiple assessments may not provide significantly more information beyond the initial assessment. Rather, a lower-stakes learning system can be used to cover a larger breadth of topics, and an additional component to weigh evidence over time is also needed.

\subsection{ACT Academy}

ACT Academy is a free online learning platform that features items and learning resources such as video lessons, games, and interactive resources that are all tagged to the HF. Users of ACT Academy are given a diagnostic report of their proficiency in all areas of the HF. They can then decide which area of the HF to study. ACT Academy then provides learning-resource recommendations based on the user's diagnostic profile and the efficacy of the learning material. The current model used in ACT Academy for computing the diagnostic profile is not the Master model but a simpler model. For details, see von Davier et al. (2019).

We obtained data from this learning system for all users who had answered items in ACT Academy from July 2014 through August 2019. The data set includes 162,093 unique users. The minimum number of items completed by a person is 1 , the maximum number is 1053 , and the median number equal to 18 . Figure 4 shows the trajectory of the posterior probability of mastery of the science skill where the horizontal axis is the number of quizzes (a set of items) that have been attempted. The color of the points in Figure 4 corresponds to the proportion of science items in the quiz answered correctly (white is all correct, black is all 


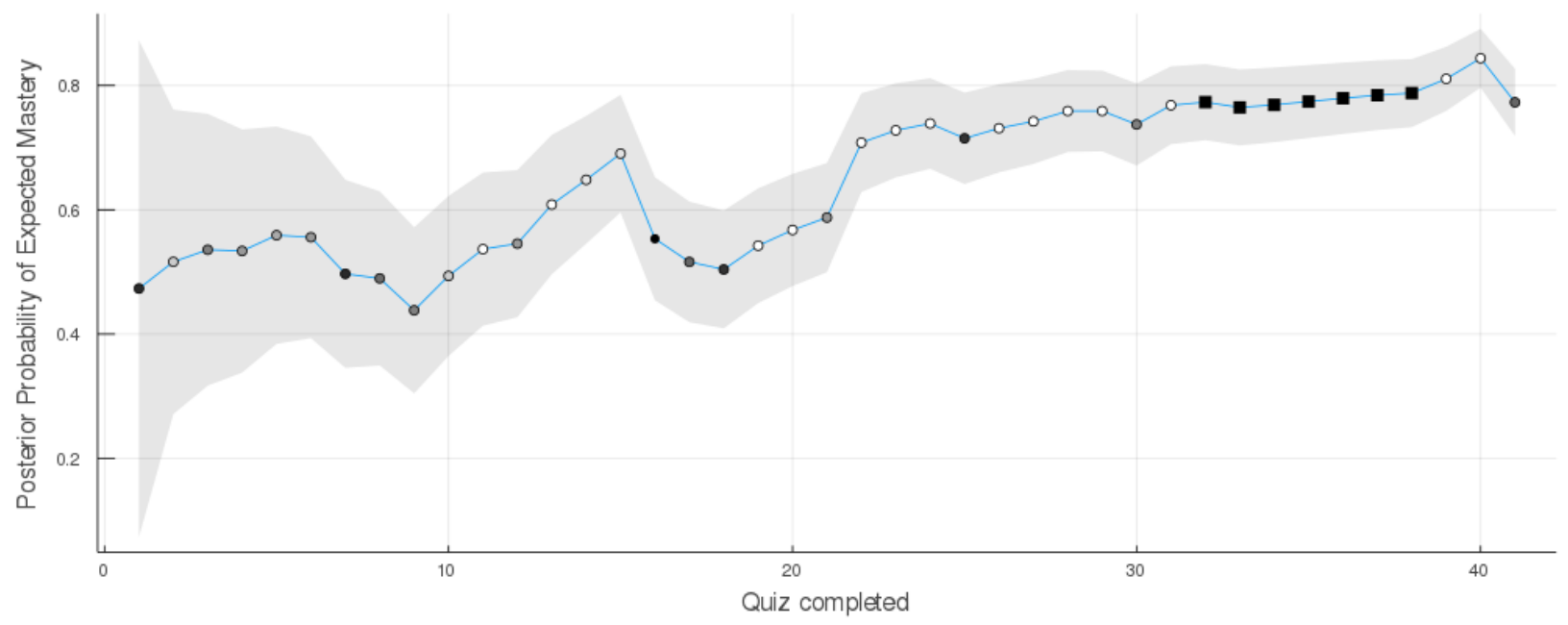

Figure 4: Trajectory of the continuously updated posterior probability of mastery of the science skill after a specific learner has completed a set of items (quiz). The color of the points indicates how many science items in the quiz were answered correctly (white is all; black is none), and square points indicate that no science items were part of the quiz.

incorrect), and square points indicate that a quiz did not contain any science items. A 95 percent confidence interval is also included. ${ }^{1}$

Figure 4 shows how the Master model can be used to track the assessment of a person's mastery of skills based on the evidence provided by their responses to items. Each item response, regardless of which skill the item is tagged to, will affect the posterior probability of mastery for every skill. The impact an item response has will be larger for skills that are more proximal to the item's tagged skill than to more distal skills. Recall, however, that since the evidence accumulates linearly, the effect of past evidence is equivalent to that of present evidence. This may be inappropriate if the underlying latent skill is changing over time. In the next section, we propose a modification to the Master model that allows us to treat past evidence differently, along with several other modifications that make the Master model better suited for use in a learning system.

\section{Extending the Master Model to Learning and Navigation}

We have shown how the Master model can be used in the context of assessment. Several aspects of this model make it well suited to a learning and navigation system as well. In particular, the way evidence accumulates to update the posterior mastery probabilities through BP provides a computationally efficient method for tracking changes in posterior mastery probability that scales well to the large number of users and user interactions in learning systems. The diagnostics provided by the Master model in the form of the

\footnotetext{
${ }^{1}$ The variance of the mastery of skill $j$ for person $p$ is given by $\tilde{\sigma}_{p j}\left(1-\tilde{\sigma}_{p j}\right)$. The confidence interval presented in Figure 4 is the point estimate of mastery \pm 1.96 times square root of the variance divided by the cumulative number of items answered.
} 


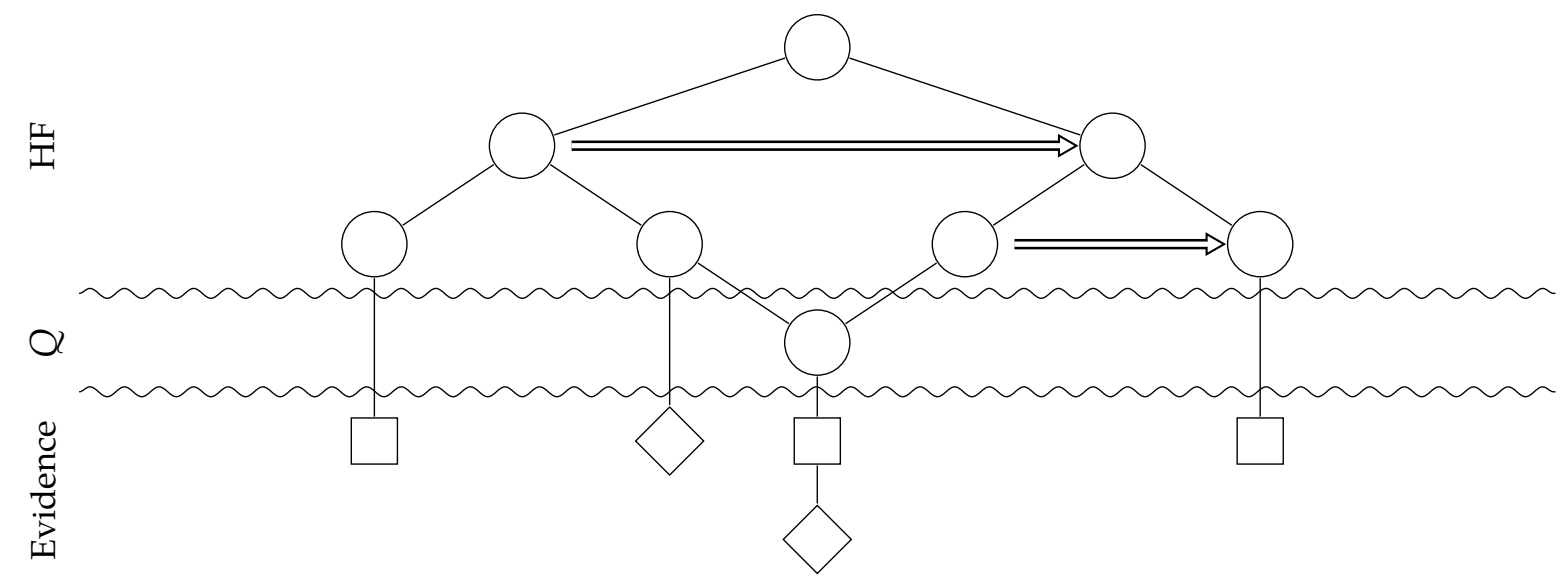

Figure 5: The network at the core of the Master model. The circles represent the skills in the holistic framework, the boxes represent items or resources that are tagged to the holistic framework. Extensions discussion in section 6.3 are also shown. The portion denoted by the $Q$ refers to complex items which are tagged to multiple skills. Arrows in the holistic framework indicate prerequisite relationships. Squares indicate items and diamonds learning resources.

posterior mastery probabilities, along with some kind of goal set by the user, also allows a learning system to provide navigation to the user by allowing recommender systems to utilize these diagnostics in order to recommend personalized learning resources, remediation, and items that are targeted to the user.

The Master model as presented above can be extended to provide additional benefits when used in a learning system context. These benefits include incorporating forgetting curves, other sources of information, ability to measure the efficacy of learning resources that are tagged to the hierarchical framework, and the inclusion of prerequisite skills or learning progressions. Some of these extensions are illustrated in Figure 5.

\subsection{Learning Over Time}

Equation 20 showed that the evidence in the Master model accumulates additively and that the order of the responses is immaterial. Although appropriate in an assessment context, this assumption may be too strong in a learning context where responses to items can span a long period of time. Forgetting curves, wherein the value of evidence is assumed to decay exponentially over time, is a classical approach to modeling decay (Ebbinghaus 1913). This approach has continued to be a dominant approach in the literature (Settles and Meeder 2016).

We suggest modifying the Master model to incorporate forgetting curves by introducing an exponential decay factor into Equation 12

$$
e_{j}(t)=\left(1-\sigma_{j}\right) \sum_{i \in \mathbf{I}_{j}} e_{i}^{-} \exp \left(-\lambda_{i}\left(t-t_{i}\right)\right)+\sigma_{j} \sum_{i \in \mathbf{I}_{j}} e_{i}^{+} \exp \left(-\lambda_{i}\left(t-t_{i}\right)\right)
$$

where $\lambda_{i}$ is an item-specific decay rate, $t_{i}$ is the time at which item $i$ was answered by an individual, and $t$ is 


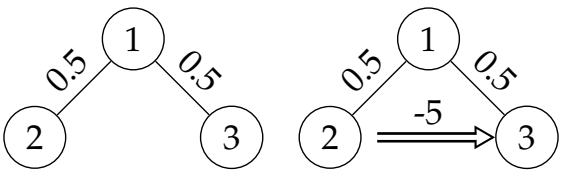

\begin{tabular}{c|cc} 
Configurations & no penalty & with penalty \\
\hline 000 & 0.26722332 & 0.3320729005 \\
001 & 0.09830597 & 0.0008231264 \\
010 & 0.09830597 & 0.1221627931 \\
011 & 0.03616474 & 0.0449411800 \\
100 & 0.03616474 & 0.0449411800 \\
101 & 0.09830597 & 0.0008231264 \\
110 & 0.09830597 & 0.1221627931 \\
111 & 0.26722332 & 0.3320729005
\end{tabular}

Figure 6: (Left) A simple network of skills, one without any pre-requisite penalties and one with (Right). The probability mass function $p(\mathbf{s})$ associated with the two networks on the left. The highlighted rows are the configurations of skill mastery that correspond to Skill 3 being mastered while Skill 2 (the prerequisite) is not mastered.

time. The evidence then accumulates as a function of time where each item's contribution to the evidence decays over time.

\subsection{Incorporating Additional Information: Pseudo-items}

Additional information that is available about a learner, such as grade point average or other demographic information, can be incorporated into the Master model through pseudo items. A pseudo item encodes additional information about a learner in the same way as an item response. Pseudo items can also be used to measure the efficacy of learning resources.

Let $z_{p l}$ be a binary variable that encodes this additional information for learner $p$ and learning resource l. For example, if $l$ is an educational video $z_{p l}=1$ would indicate that learner $p$ had watched that video. Or $z_{p l}=1$ may indicate that a learner's grade point average is in a certain range. If we assume that these additional resources or pieces of information are independent of one another and independent from the item responses (conditional on mastery of the skills), we can add the following term to the measurement model:

$$
p\left(\sigma_{p} \mid \mathbf{z}_{p}\right)=\prod_{l}\left[\gamma_{l}^{\sigma_{p j}}\left(1-\gamma_{l}\right)^{1-\sigma_{p j}}\right]^{z_{p l}}
$$

where $\sigma_{p j}$ is the latent mastery indicator for the skill to which resource $l$ is tagged and $\gamma_{l}$ is the parameter associated with information $l$ and measures its efficacy.

\subsection{Prerequisites and Complex Items}

One of the key assumptions needed to use BP to update a learner's skill mastery probabilities is that the skills are in a graphical network that takes the form of a hierarchical tree. Adding edges to the hierarchical tree will always introduce a loop. Such edges are necessary to add, however, if we wish to incorporate two necessary components into the model: prerequisites and complex items tagged to multiple skills. Adding prerequisites to the hierarchical structure of the skills allows a designer of a learning system to plot out a 
learning progression for users of the learning system.

Learning is much more than a complex list of what a student should be able to do by a certain age level as encoded by the hierarchical framework in the Master model. In the literature, two large branches of research have been established in order to provide a more principled solution to incorporate learning. One branch stems from mastery learning (Bloom 1968), while another from knowledge spaces (Doignon and Falmagne 1985). The main way that these approaches differ from a set of standards is that the path a learner takes through the materials is personalized based on what the learner is currently able to do and what the learner is capable of learning. Such ideas are incorporated into the mastery model via prerequisites or prerequisite relationships defined as relationships between skills where one skill cannot be mastered without a certain level of mastery in another skill.

Adding prerequisites requires us to add an additional term to the skill distribution:

$$
p\left(\mathbf{s}_{p}\right)=\frac{1}{Z_{p}} \exp \left(\mathbf{s}_{p}^{\top} \boldsymbol{\mu}+\frac{1}{2} \mathbf{s}_{p}^{\top} \mathbf{H} \mathbf{s}_{p}+\delta_{-}\left(\mathbf{s}_{p}\right)^{\top} \boldsymbol{\Lambda} \delta_{+}\left(\mathbf{s}_{p}\right)\right)
$$

where $\delta_{x}\left(\mathbf{s}_{p}\right)=\left(\mathbf{1}\left\{s_{p 1}=x\right\}, \ldots, \mathbf{1}\left\{s_{p r}=x\right\}\right)^{\top}$ is the Dirac delta function and $\boldsymbol{\Lambda}$ is a matrix of penalty values where $\lambda_{j k}$ is the penalty value associated with skill configurations in which skill $k$ is mastered while the prerequisite skill $j$ is not. Consider, for example, the simple skill network of the motivating example. Suppose $\tau=0.5, h_{j k}=1$ for all $j$ and $k$, and $\lambda_{23}=-5$. Figure 6 illustrates the change in the prior distribution $p(\mathbf{s})$ when the penalty term is included. Note that the highlighted entries in the table correspond to configurations in which Skill 3 is mastered while Skill 2 (the prerequisite) is not. The probability in these highlighted configurations is lower when including the penalty term than when excluding the penalty term, indicating how the penalty term can be used to modify the prior distribution of skills. Such modifications can lead to better routing of students through material tagged to these skills by leveraging the known prerequisite structure among the skills.

How does this change in the prior impact the posterior probability of mastery? Consider 10 items tagged to the small example in Figure 6. The first three items are tagged to Skill 2, and the other seven are tagged to Skill 3. We sample the $\alpha$ parameters independently and uniformly between 0.34 and 0.45 and the $\beta$ independently and uniformly between 0.55 and 0.65 . We assume that the three items tagged to Skill 2 are answered correctly and loop through all $2^{7}$ possible response patterns for the remaining items tagged to Skill 3. Figure 7 shows the median of the differences in posterior probability of mastery of Skill 3 between the skill model with and without the penalty terms. The facets are varying values of $\tau(0.0,0.5$, and 1.0). The horizontal axis shows increasing values of the penalty $\lambda_{23}$ (from 0 to 4 ). The varying response patterns are grouped by their sum scores and colored respectively. Figure 7 shows that the difference in the posterior probability of mastering skill 3 increases when the penalty increases. This effect is modulated by the magnitude of $\tau$ and the edge-weights $h_{j k}$, as well as by the amount of evidence the item responses provide. If a person answers all 10 items (in)correctly, the model infers that she has (not) mastered Skill 3 - 

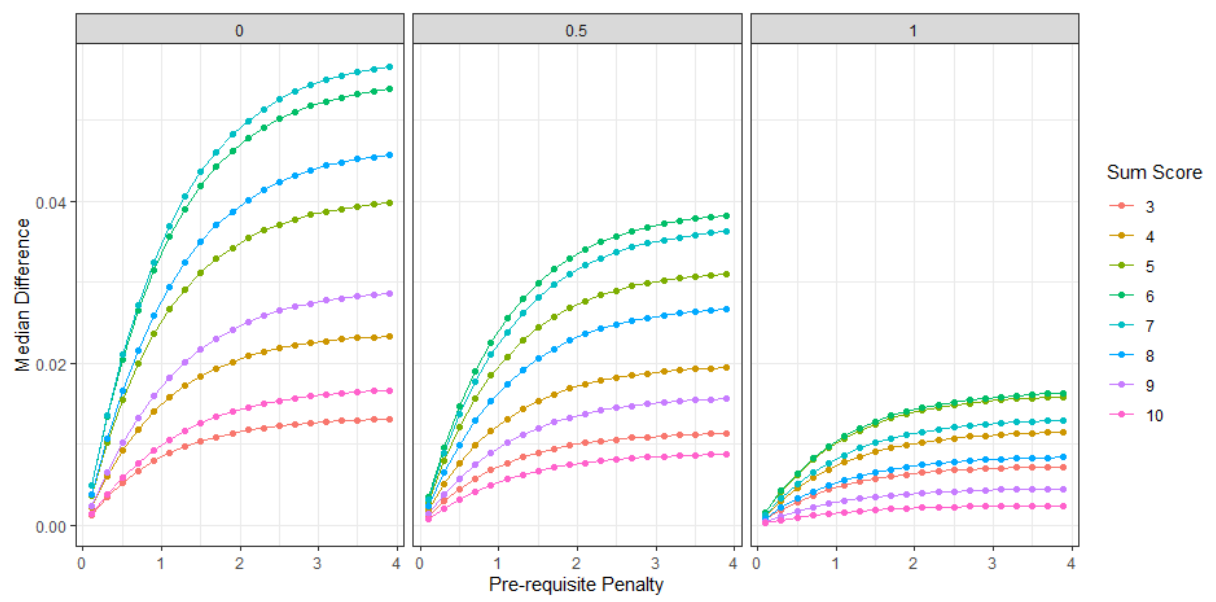

Figure 7: Median difference in posterior probability of mastery of skill 3 from Figure 6. Facets correspond to varying values of $\tau$, horizontal axis is the penalty parameter, $\lambda_{23}$, and response patterns are grouped by sum score and colored respectively.

regardless of the presence of the penalty term in the prior. Barring these extreme response patterns, the use of the penalty term in the prior does affect the posterior probability of mastery.

Adding prerequisites to the model is not the only way that edges can be added to the skill network. Adding complex items tagged to multiple skills does this as well. Adding complex items requires a more complex measurement model such as the G-DINA (de la Torre 2011), which allows for multiple skills to be required for an item through a construct known as the $Q$ matrix (Tatsuoka 1983). Both of these extensions add more edges to the graphical structure of the Bayesian network associated with the Master model, preventing us from using BP to obtain exact expectations. There are two possible solutions to this: loopy belief propagation and the junction tree algorithm. The standard BP algorithm involves sending information from the leaves of the tree up to the root and then passing updated information about masteries back down from the root to the leaves - totalling two passes through all of the skills. When there are loops in the tree, research has shown that the marginal distributions can be approximated well in most cases by simply running this algorithm several more times (Weiss 2000). This is known as loopy belief propagation. The alternative is to use the junction tree algorithm. This algorithm will again provide us with exact marginals but at the expense of much longer computation time. It entails constructing a new acyclic graph, or a graph without cycles, by clustering nodes in the original graph. Such a graph is called a junction graph. Once this graph has been constructed, standard BP can be performed since the junction graph is guaranteed to not have cycles. 


\section{$7 \quad$ Future Work and Conclusion}

In this paper, we have discussed a new statistical model for learning, assessment, and navigation; explained its origins and estimation; and provided an example from real data. The Master model can be seen as a way to combine the evidence that is provided by item responses to make judgements about skill mastery. Although initially developed for a holistic framework, the model is "agnostic" to the framework used and not restricted to any particular learning platform. In this way, the current study aimed to lay a foundation for applied research capable of helping educators and educational institutions globally achieve their goal of helping people to learn.

We have outlined several ways to extend the Master model to broaden its application. There are three additional possibilities that are worth mentioning as topics for future research. Throughout this paper we have assumed that the structure of the skill network, that is, the edges between the skills for which $h_{j k} \neq 0$, was known. An opportune extension of the work presented here would be to jointly estimate the structure of this network along with the parameters of the Master model. The purpose would be to provide test designers with empirical feedback on the validity of the postulated network and suggest data-driven augmentations to it. Several approaches for learning latent tree graphical models from data have been proposed in the literature (Choi et al. 2011; Parikh et al. 2011). A second extension deals with situations where items are tagged to multiple frameworks. We'll take the ACT as an example. The English items in the test belong to passages. It is not uncommon for items from the same passage to correlate more with one another than with items from a different passage. Such dependencies, which exist over and above those implied by the underlying skills, can be taken into account by adding a second hierarchy to the model. An item relates to a skill and also to a passage. A third natural extension deals with evidence that is not item responses. This entails changing the measurement part of the model. We only need to adapt the distribution of evidence given (non)mastery, while all the rest of the model stays intact. This allows for incorporating evidence from response times, eye tracking, pupil dilation, etc. 


\section{References}

Almond, R. G., Mislevy, R. J., Steinberg, L. S., Yan, D., and Williamson, D. M. (2015). Bayesian networks in educational assessment. Springer.

Bloom, B. S. (1968). “Learning For Mastery: Instruction and curriculum”. In: Comment Evaluation UCLA-CSIEP 1.2, pp. 1-12.

Borsboom, D. (2008). "Psychometric perspectives on diagnostic systems". In: Journal of Clinical Psychology 64.9, pp. 1089-1108. eprint: https : //onlinelibrary . wiley . com/doi/pdf/10 . $1002 / \mathrm{jclp} .20503$.

Camara, W., O'Connor, R., Mattern, K., and Hanson, M. A. (2015). Beyond Academics: A Holistic Framework for Enhancing Education and Workplace Success. ACT Research Report Series 4. ACT.

Carroll, J. B. (1993). Human Cognitive Abilities: A Survey of Factor-Analytic Studies. Cambridge University Press.

Celeux, G. and Diebolt, J. (1992). "A stochastic approximation type EM algorithm for the mixture problem". In: Stochastics and Stochastic Reports 41.1-2, pp. 119-134. eprint: https : //doi.org/10.1080/17442509208833797.

Choi, M. J., Tan, V. Y. F., Anandkumar, A., and Willsky, A. S. (May 2011). “Learning Latent Tree Graphical Models". In: Journal of Machine Learning Research 12, pp. 1771-1812.

Cronbach, L. J. (Nov. 1957). "The two disciplines of scientific psychology". In: American Psychologist 12.11, pp. 671-684.

De la Torre, J. (Apr. 2011). “The Generalized DINA Model Framework”. In: Psychometrika 76.2, pp. 179-199.

Dempster, A. P., Laird, N. M., and Rubin, D. B. (1977). “Maximum Likelihood from Incomplete Data via the EM Algorithm". In: Journal of the Royal Statistical Society B 39.1, pp. 1-38.

Deonovic, B., Yudelson, M., Bolsinova, M., Attali, M., and Maris, G. (Oct. 2018). “Learning meets assessment". In: Behaviormetrika 45.2, pp. 457-474.

Doignon, J.-P. and Falmagne, J.-C. (1985). "Spaces for the assessment of knowledge". In: International Journal of Man-Machine Studies 23.2, pp. 175-196.

Ebbinghaus, H. (1885). Über das Gedächtnis: Untersuchungen zur experimentellen Psychologie. Leipzig: Duncker \& Humblot.

Ebbinghaus, H. (1913). Memory: A Contribution to Experimental Psychology. Trans. by H. A. Ruger and C. E. Bussenius. English Translation of Ebbinghaus (1885). New York: Teachers College, Columbia University. 
Embretson, S. and Gorin, J. S. (2001). “Improving Construct Validity With Cognitive Psychology Principles". In: Journal of Educational Measurement 38.4, pp. 343-368.

Epskamp, S. (2017). “Network Psychometrics”. PhD thesis. University of Amsterdam.

Gallager, R. G. (1963). Low-Density Parity-Check Codes. Cambridge, MA, USA: MIT Press.

Gorin, J. S. (2006). “Test Design with Cognition in Mind”. In: Educational Measurement: Issues and Practice 25.4, pp. 21-35.

Griffiths, R. B. (1967). “Correlations in Ising ferromagnets. III. A mean-field bound for binary correlations". In: Comm. Math. Phys. 6.2, pp. 121-127.

Hannon, B. (Oct. 2014). "Predicting College Success: The Relative Contributions of Five Social/Personality Factors, Five Cognitive/Learning Factors, and SAT Scores". In: Journal of Education and Training Studies 2.4, pp. 46-58.

Hunter, D. and Lange, K. (2004). "A Tutorial on MM Algorithms". In: American Statistician 58, Issue 1, pp. 30-37.

Ising, E. (Feb. 1925). “Beitrag zur Theorie des Ferromagnetismus". In: Zeitschrift für Physik 31.1, pp. 253-258.

Lenz, W. (1920). “Beitrag zum Verständnis der magnetischen Erscheinungen in festen Körpern”. In: Z. Phys. 21, pp. 613-615.

Maris, G., Bechger, T., and van Ruitenburg, J. (2014). “Minorization Algorithms in Item Response Theory". In: Psychology and Statistics: Ready for the Future?: Liber Amicorum for Willem J Heiser. Ed. by M. de Rooij and C. M. van Putten. Leiden University Press.

Marsman, M., Borsboom, D., Kruis, J., Epskamp, S., van Bork, R., Waldorp, L., van der Maas, H., and Maris, G. (2018). "An introduction to network psychometrics: Relating ising network models to item response theory models". In: Multivariate behavioral research 53.1, pp. 15-35.

Marsman, M., Maris, G., Bechger, T., and Glas, C. (2015). “Bayesian inference for low-rank Ising networks". In: Scientific Reports 5.1.

McEliece, R. J., MacKay, D. J. C., and Jung-Fu Cheng (1998). “Turbo decoding as an instance of Pearl's "belief propagation" algorithm". In: IEEE Journal on Selected Areas in Communications 16.2, pp. $140-152$.

Nakanishi, K. (Apr. 1981). “Two- and three-spin cluster theory of spin-glasses". In: Phys. Rev. B 23 (7), pp. 3514-3522.

National Research Council (2001). Knowing What Students Know: The Science and Design of Educational Assessment. Ed. by J. W. Pellegrino, N. Chudowsky, and R. Glaser. Washington, DC: The National Academies Press. 
Parikh, A. P., Song, L., and Xing, E. P. (2011). “A Spectral Algorithm for Latent Tree Graphical Models". In: Proceedings of the 28th International Conference on International Conference on Machine Learning. ICML'11. Bellevue, Washington, USA: Omnipress, pp. 1065-1072.

Pearl, J. (1988). Probabilistic Reasoning in Intelligent Systems: Networks of Plausible Inference. San Francisco, CA, USA: Morgan Kaufmann Publishers Inc.

Pellegrino, J. W., Baxter, G. P., and Glaser, R. (1999). “Chapter 9: Addressing the “Two Disciplines" Problem: Linking Theories of Cognition and Learning With Assessment and Instructional Practice". In: Review of research in education 24.1, pp. 307-353.

Rupp, A. A., Templin, J., and Henson, R. A. (2010). Diagnostic measurement: Theory, methods, and applications. Guilford Press.

Savi, A. O., Marsman, M., van der Maas, H., and Maris, G. (2019). "The Wiring of Intelligence". In: Perspectives on Psychological Science 14.6. PMID: 31647746, pp. 1034-1061. eprint: https: //doi.org/10.1177/1745691619866447.

Savi, A. O., van der Maas, H., and Maris, G. (Feb. 2015). “Navigating Massive Open Online Courses". English. In: Science 347.6225, pp. 958-958.

Settles, B. and Meeder, B. (2016). "A trainable spaced repetition model for language learning". In: Proceedings of the 54th annual meeting of the association for computational linguistics (volume 1: long papers), pp. $1848-1858$.

Spearman, C. (1904). “"General Intelligence," objectively determined and measured". In: The American Journal of Psychology 15.2, pp. 201-292.

Stewart, S. B. (2015). “Grit and Self-Control as Predictors of First-Year Student Success". PhD thesis. University of Southern Maine.

Tatsuoka, K. K. (1983). “Rule space: An approach for dealing with misconceptions based on item response theory". In: Journal of educational measurement 20.4, pp. 345-354.

Undheim, J. O. and Gustafsson, J.-E. (1987). “The Hierarchical Organization of Cognitive Abilities: Restoring General Intelligence Through the Use of Linear Structural Relations (LISREL)". In: Multivariate Behavioral Research 22.2. PMID: 26782063, pp. 149-171. eprint: https ://doi .org/ 10.1207/s15327906mbr2202_2.

Van der Maas, H., Dolan, C. V., Grasman, R. P., Wicherts, J. M., Huizenga, H. M., and Raijmakers, M. E. (2006). “A dynamical model of general intelligence: the positive manifold of intelligence by mutualism." In: Psychological review 113.4, p. 842. 
Von Davier, A. A., Deonovic, B., Yudelson, M., Polyak, S. T., and Woo, A. (2019). “Computational Psychometrics Approach to Holistic Learning and Assessment Systems". In: Frontiers in Education 4, p. 69.

Wei, G. C. G. and Tanner, M. A. (1990). “A Monte Carlo Implementation of the EM Algorithm and the Poor Man's Data Augmentation Algorithms". In: Journal of the American Statistical Association 85.411, pp. 699-704. eprint: https://www . tandfonline.com/doi/pdf/10 . 1080/ Q1621459.1990.10474930.

Weiss, Y. (Jan. 2000). “Correctness of Local Probability Propagation in Graphical Models with Loops". In: Neural Computation 12.1, pp. 1-41. 


\section{A Derivations of $\log$ posterior odds}

When direct evidence is taken into account, the marginal posterior that skill $j$ is mastered is:

$$
\begin{aligned}
p\left(s_{j}=+1 \mid \mathbf{x}\right) & =\sum_{\mathbf{s}: s_{j}=+1} p(\mathbf{s} \mid \mathbf{x}) \\
& =\frac{1}{Z_{\mathbf{x}}} \sum_{\mathbf{s}: s_{j}=+1} \exp \left(\sum_{j} e_{j}+\mathbf{s}^{\top} \boldsymbol{\mu}+\frac{\tau}{2} \mathbf{s}^{\top} \mathbf{H} \mathbf{s}\right) \\
& =\frac{1}{Z_{\mathbf{x}}} \exp \left(\sum_{i \in \mathbf{I}_{j}} e_{i}^{+}\right) \sum_{\mathbf{s}: s_{j}=+1} \exp \left(\sum_{k \neq j} e_{k}^{*}+\mathbf{s}^{\top} \boldsymbol{\mu}+\frac{\tau}{2} \mathbf{s}^{\top} \mathbf{H s}\right) \\
& =\exp \left(\sum_{i \in \mathbf{I}_{j}} e_{i}^{+}\right) \frac{Z_{\mathbf{x}_{-j}}^{+}}{Z_{\mathbf{x}}}
\end{aligned}
$$

A similar expression can be derived for the posterior of non-mastery. It follows that:

$$
\begin{aligned}
\log \frac{p\left(s_{j}=+1 \mid \mathbf{x}\right)}{p\left(s_{j}=-1 \mid \mathbf{x}\right)} & =\sum_{i \in \mathbf{I}_{j}}\left(e_{i}^{+}-e_{i}^{-}\right)+\log Z_{\mathbf{x}_{-j}}^{+}-\log Z_{\mathbf{x}_{-j}}^{-} \\
& =\sum_{i \in \mathbf{I}_{j}}\left(e_{i}^{+}-e_{i}^{-}\right)+\log \left(\frac{\pi_{j}^{*}}{1-\pi_{j}^{*}}\right) \\
& =\sum_{i \in \mathbf{I}_{j}} x_{i} \log \left(\frac{\beta_{i}}{\alpha_{i}}\right)+\left(1-x_{i}\right) \log \left(\frac{1-\beta_{i}}{1-\alpha_{i}}\right)+\log \left(\frac{\pi_{j}^{*}}{1-\pi_{j}^{*}}\right) \\
& =\sum_{i \in \mathbf{I}_{j}} w_{i}+\log \left(\frac{\pi_{j}^{*}}{1-\pi_{j}^{*}}\right)
\end{aligned}
$$

\section{B Derivation of Expectation Maximization Estimates}

\section{B.1 Estimating $\alpha$ and $\beta$}

$$
\begin{aligned}
\frac{\partial Q\left(\boldsymbol{\theta}, \boldsymbol{\theta}^{(t)}\right)}{\partial \alpha_{i}} & =\sum_{p=1}^{n} \frac{\partial}{\partial \alpha_{i}}\left(\log \alpha_{i}\right)\left(1-\tilde{\sigma}_{p j_{i}}\right) x_{p i}+\frac{\partial}{\partial \alpha_{i}}\left(\log \left(1-\alpha_{i}\right)\right)\left(1-\tilde{\sigma}_{p j_{i}}\right)\left(1-x_{p i}\right) \\
0 & \stackrel{\text { set }}{=} \sum_{p=1}^{n} \frac{\left(1-\tilde{\sigma}_{p j_{i}}\right) x_{p i}}{\alpha_{i}}-\frac{\left(1-\tilde{\sigma}_{p j_{i}}\right)\left(1-x_{p i}\right)}{1-\alpha_{i}} \\
& =\sum_{p=1}^{n} \frac{\left(1-\tilde{\sigma}_{p j_{i}}\right) x_{p i}-\alpha_{i}\left(1-\tilde{\sigma}_{p j_{i}}\right)}{\alpha_{i}\left(1-\alpha_{i}\right)} \\
\alpha_{i} & =\frac{\sum_{p=1}^{n}\left(1-\tilde{\sigma}_{p j_{i}}\right) x_{p i}}{\sum_{p=1}^{n}\left(1-\tilde{\sigma}_{p j_{i}}\right)}
\end{aligned}
$$


and similarly for $\beta_{i}$ we get

$$
\beta_{i}=\frac{\sum_{p=1}^{n} \tilde{\sigma}_{p j_{i}} x_{p i}}{\sum_{p=1}^{n} \tilde{\sigma}_{p j_{i}}}
$$

\section{B.2 Inverse temperature $\tau$ and external magnetic field $\mu$}

For the temperature we get

$$
\frac{\partial Q\left(\boldsymbol{\theta}, \boldsymbol{\theta}^{(t)}\right)}{\partial \tau}=\sum_{p=1}^{n} \frac{1}{2} E_{S \mid \mathbf{x}, \boldsymbol{\theta}^{(t)}}\left[\mathbf{s}_{p}^{\top} \mathbf{H} \mathbf{s}_{p}\right]-\sum_{p=1}^{n} \frac{\partial}{\partial \tau} \log Z_{S}
$$

the last term is given by

$$
\begin{aligned}
\frac{\partial}{\partial \tau} \log Z_{S} & =\frac{\frac{\partial}{\partial \tau} Z_{S}}{Z_{S}} \\
& =\frac{1}{Z_{S}} \sum_{S} \frac{1}{2} \mathbf{s}^{\top} \mathbf{H} \mathbf{s} \exp \left(\mu^{\top} \mathbf{s}+\frac{\tau}{2} \mathbf{s}^{\top} \mathbf{H} \mathbf{s}\right) \\
& =E_{S \mid \mu, \tau}\left[\frac{1}{2} \mathbf{s}^{\top} \mathbf{H} \mathbf{s}\right]
\end{aligned}
$$

Note the last expectation is with respect to $S$ and not the conditional distribution $S \mid \mathbf{X}$. Furthermore, the last expectation is also a function of $\tau$. So the $\tau$ which maximizes the $Q$ is the one for which

$$
\frac{1}{n} \sum_{p=1}^{n} E_{S \mid \mathbf{x}, \boldsymbol{\theta}^{(t)}}\left[\frac{1}{2} \mathbf{s}_{p}^{\top} \mathbf{H s}_{p}\right]=\mathrm{E}_{S \mid \mu, \tau}\left[\frac{1}{2} \mathbf{s}^{\top} \mathbf{H} \mathbf{s}\right]
$$

For $\mu$ we have

$$
\frac{\partial Q\left(\boldsymbol{\theta}, \boldsymbol{\theta}^{(t)}\right)}{\partial \mu}=\sum_{p=1}^{n} E_{S \mid \mathbf{x}, \boldsymbol{\theta}^{(t)}}\left[\mathbf{s}_{p}\right]-\sum_{p=1}^{n} \frac{\partial}{\partial \mu} \log Z_{S}
$$

the last term is given by

$$
\begin{aligned}
\frac{\partial}{\partial \mu} \log Z_{S} & =\frac{\frac{\partial}{\partial \mu} Z_{S}}{Z_{S}} \\
& =\frac{1}{Z_{S}} \sum_{S} \mathbf{s} \exp \left(\mu^{\top} \mathbf{s}+\frac{\tau}{2} \mathbf{s}^{\top} \mathbf{H} \mathbf{s}\right) \\
& =E_{S \mid \mu, \tau}[\mathbf{s}]
\end{aligned}
$$

As above the last expectation is a function with respect to $\mu$ and so the $\mu$ which maximizes $Q$ is one where

$$
\frac{1}{n} \sum_{p=1}^{n} E_{S \mid \mathbf{x}, \boldsymbol{\theta}^{(t)}}\left[\mathbf{s}_{p}\right]=\mathrm{E}_{S \mid \boldsymbol{\mu}, \tau}[\mathbf{s}]
$$


Thus, we don't have a closed form solution for neither $\tau$ nor $\mu$. We can use an iterative procedure such as Newton-Raphson. Define

$$
f(\tau)=\frac{1}{n} \sum_{p=1}^{n} E_{S \mid \mathbf{x}, \boldsymbol{\theta}^{(t)}}\left[\frac{1}{2} \mathbf{s}_{p}^{\top} \mathbf{H} \mathbf{s}_{p}\right]-\mathrm{E}_{S \mid \mu, \tau}\left[\frac{1}{2} \mathbf{s}^{\top} \mathbf{H} \mathbf{s}\right]
$$

Let $\tau_{k}$ and $\mu_{k}$ be the value of the estimates at the $k$ th iteration of the Newton-Raphson algorithm. The Newton-Raphson solution is obtained by iterating

$$
\tau_{k+1}=\tau_{k}-f\left(\tau_{k}\right) / f^{\prime}\left(\tau_{k}\right)
$$

The derivative is given by

$$
\begin{aligned}
f^{\prime}(\tau) & =-\frac{\partial}{\partial \tau} E_{S \mid \mu_{k}, \tau_{k}}\left[\frac{1}{2} \mathbf{s}^{\top} \mathbf{H s}\right] \\
& =-\frac{\partial}{\partial \tau} \frac{\frac{\partial}{\partial \tau} Z_{S}}{Z_{S}} \\
& =-\left(\frac{\frac{\partial^{2}}{\partial \tau^{2}} Z_{S}}{Z_{S}}-\left(\frac{\frac{\partial}{\partial \tau} Z_{S}}{Z_{S}}\right)^{2}\right)
\end{aligned}
$$

the second derivative of the normalizing constant is

$$
\frac{\partial^{2}}{\partial \tau^{2}} Z_{S}=\sum_{S}\left(\frac{1}{2} \mathbf{s}^{\top} \mathbf{H} \mathbf{s}\right)^{2} \exp \left(\mu^{\top} \mathbf{s}_{p}+\frac{\tau}{2} \mathbf{s}^{\top} \mathbf{H} \mathbf{s}\right)
$$

Plugging into Equation 63 we have

$$
\begin{aligned}
& =-\left(\mathrm{E}_{S \mid \mu_{k}, \tau_{k}}\left[\left(\frac{1}{2} \mathbf{s}^{\top} \mathbf{H} \mathbf{s}\right)^{2}\right]-\mathrm{E}_{S \mid \mu_{k}, \tau_{k}}\left[\frac{1}{2} \mathbf{s}^{\top} \mathbf{H} \mathbf{s}\right]^{2}\right) \\
& =-\operatorname{Var}_{S \mid \mu_{k}, \tau_{k}}\left[\frac{1}{2} \mathbf{s}^{\top} \mathbf{H s}\right]
\end{aligned}
$$

So the Newton-Raphson iteration is given by

$$
\tau_{k+1}=\tau_{k}+\frac{\frac{1}{n} \sum_{p=1}^{n} E_{S \mid \mathbf{x}, \boldsymbol{\theta}^{(t)}}\left[\frac{1}{2} \mathbf{s}_{p}^{\top} \mathbf{H} \mathbf{s}_{p}\right]-\mathrm{E}_{S \mid \boldsymbol{\mu}_{k}, \tau_{k}}\left[\frac{1}{2} \mathbf{s}^{\top} \mathbf{H} \mathbf{s}\right]}{\operatorname{Var}_{S \mid \mu_{k}, \tau_{k}}\left[\frac{1}{2} \mathbf{s}^{\top} \mathbf{H} \mathbf{s}\right]}
$$

Similarly for $\mu$ we get

$$
\mu_{k+1}=\mu_{k}+\operatorname{Cov}_{S \mid \mu_{k}, \tau_{k}}[\mathbf{s}]^{-1}\left(\frac{1}{n} \sum_{p=1}^{n} E_{S \mid \mathbf{x}, \boldsymbol{\theta}^{(t)}}\left[\mathbf{s}_{p}\right]-\mathrm{E}_{S \mid \mu_{k}, \tau_{k}}[\mathbf{s}]\right)
$$


Consider starting the Newton-Raphson iterations with $\tau_{0}=0$ and $\mu_{0}=\mathbf{0}$. In this case

$$
\begin{aligned}
\mathrm{E}_{S \mid \mu_{k}, \tau_{k}}\left[\mathbf{s}^{\top} \mathbf{H} \mathbf{s}\right] & =0 \\
\operatorname{Var}_{S \mid \mu_{k}, \tau_{k}}\left[\frac{1}{2} \mathbf{s}^{\top} \mathbf{H s}\right] & =\frac{1}{2} \sum_{j_{1} \neq j_{2}} h_{j_{1} j_{2}}^{2} \\
\mathrm{E}_{S \mid \mu_{k}, \tau_{k}}[\mathbf{s}] & =\mathbf{0} \\
\operatorname{Cov}_{S \mid \mu_{k}, \tau_{k}}[\mathbf{s}] & =\mathbf{1}
\end{aligned}
$$

If we perform only one iteration of the Newton-Raphson we then get

$$
\begin{aligned}
& \tau=\frac{1}{n} \sum_{p=1}^{n} E_{S \mid \mathbf{x}, \boldsymbol{\theta}^{(t)}}\left[\frac{1}{2} \mathbf{s}_{p}^{\top} \mathbf{H} \mathbf{s}_{p}\right] /\left(\frac{1}{2} \sum_{j_{1} \neq j_{2}} h_{j_{1} j_{2}}^{2}\right) \\
& \mu=\frac{1}{n} \sum_{p=1}^{n} E_{S \mid \mathbf{x}, \boldsymbol{\theta}^{(t)}}\left[\mathbf{s}_{p}\right]
\end{aligned}
$$

\section{B.3 Estimating the Connectivity Matrix $H$}

In the above sections we assumed that the values of $\mathbf{H}$ were known. We can relax this assumption. Suppose that the indices for which $h_{j_{1} j_{2}}=0$ were known, but we want to freely estimate $h_{j_{1} j_{2}} \neq 0$.

$$
\frac{\partial Q\left(\boldsymbol{\theta}, \boldsymbol{\theta}^{(t)}\right)}{\partial h_{j_{1} j_{2}}}=\sum_{p=1}^{n} \tau E_{S \mid \mathbf{x}, \boldsymbol{\theta}^{(t)}}\left[s_{p j_{1}} s_{p j_{2}}\right]-\sum_{p=1}^{n} \frac{\partial}{\partial h_{j_{1} j_{2}}} \log Z_{S}
$$

the last term is given by

$$
\begin{aligned}
\frac{\partial}{\partial h_{j_{1} j_{2}}} \log Z_{S} & =\frac{\frac{\partial}{\partial h_{j_{1} j_{2}}} Z_{S}}{Z_{S}} \\
& =\frac{1}{Z_{S}} \sum_{S} \tau s_{j_{1}} s_{j_{2}} \exp \left(\mu^{\top} \mathbf{s}+\frac{\tau}{2} \mathbf{s}^{\top} \mathbf{H} \mathbf{s}\right) \\
& =\tau \mathrm{E}_{S \mid \mu, \tau}\left[s_{j_{1}} s_{j_{2}}\right]
\end{aligned}
$$

and so the $h_{j_{1} j_{2}}$ which maximizes $Q$ is one where

$$
\frac{1}{n} \sum_{p=1}^{n} E_{S \mid \mathbf{x}, \boldsymbol{\theta}^{(t)}}\left[s_{p j_{1}} s_{p j_{2}}\right]=\mathrm{E}_{S \mid \boldsymbol{\mu}, \tau}\left[s_{j_{1} j_{2}}\right]
$$

If $\mathbf{H}$ is a a tree structure and $\boldsymbol{\mu}=\mathbf{0}$ then it has been shown (Griffiths 1967) that $\mathrm{E}_{S \mid \mu, \tau}\left[s_{j_{1} j_{2}}\right]=\tanh \left(\tau h_{j_{1} j_{2}}\right)$ so the final estimator becomes

$$
h_{j_{1} j_{2}}=\frac{1}{\tau} \operatorname{atanh}\left(\frac{1}{n} \sum_{p=1}^{n} E_{S \mid \mathbf{x}, \boldsymbol{\theta}^{(t)}}\left[s_{p j_{1}} s_{p j_{2}}\right]\right)
$$

\section{Notation}




\begin{tabular}{|c|c|c|}
\hline Notation & Meaning & $\begin{array}{l}\text { Possible Values } \\
\text { for Elements }\end{array}$ \\
\hline$n$ & number of respondents & \\
\hline$m_{p}, m$ & $\begin{array}{l}\text { number of items responded to by respondent } \\
p \text {, and the total number of items }\end{array}$ & \\
\hline$r$ & number of skills in hierarchy & \\
\hline $\mathbf{x}_{p}=\left(x_{p 1}, \ldots, x_{p m_{p}}\right)^{\top}$ & $\begin{array}{l}\text { responses of individual } p \text { to a subset of size } \\
m_{p} \text { of all of the } m \text { items. }\end{array}$ & $\{0,1\}$ \\
\hline $\mathbf{i}_{p}=\left(i_{p 1}, \ldots, i_{p m_{p}}\right)^{\top}$ & $\begin{array}{l}\text { the index of items which person } p \text { answered, } \\
\mathbf{i}_{p} \subseteq\{1, \ldots, m\}\end{array}$ & $\{1, \ldots, m\}$ \\
\hline $\mathbf{j}=\left(j_{1}, \ldots, j_{m}\right)^{\top}$ & the index of skills tagged to each item. & $\{1, \ldots, k\}$ \\
\hline $\mathbf{s}_{p}=\left(s_{p 1}, \ldots, s_{p r}\right)^{\top}$ & $\begin{array}{l}\text { the random vector of mastery of the } k \text { skills } \\
\text { for individual } p\end{array}$ & $\{-1,+1\}$ \\
\hline $\boldsymbol{\sigma}_{p}=\left(\sigma_{p 1}, \ldots, \sigma_{p r}\right)^{\top}$ & same as $\mathbf{s}_{p}$ but transformed so $s_{p j}=2 \sigma_{p j}-1$ & $\{0,1\}$ \\
\hline$\tilde{\boldsymbol{\sigma}}_{p}=\left(\tilde{\sigma}_{p 1}, \ldots, \tilde{\sigma}_{p r}\right)^{\top}$ & $\begin{array}{l}\text { expected mastery of person } p \text { for skills, } \tilde{\sigma}_{p j}= \\
E_{S \mid \mathbf{x}, \boldsymbol{\theta}^{(t)}}\left[\sigma_{p j}\right]\end{array}$ & $(0,1)$ \\
\hline$\alpha=\left(\alpha_{1}, \ldots, \alpha_{m}\right)^{\top}$ & $\begin{array}{l}\text { probability of a non-master of skill } j_{i} \text { answer- } \\
\text { ing item } i \text { correctly }\end{array}$ & $(0,1)$ \\
\hline $\boldsymbol{\beta}=\left(\beta_{1}, \ldots, \beta_{m}\right)^{\top}$ & $\begin{array}{l}\text { probability of a master of skill } j_{i} \text { answering } \\
\text { item } i \text { correctly }\end{array}$ & $(0,1)$ \\
\hline$\mu=\left(\mu_{1}, \ldots, \mu_{r}\right)^{\top}$ & $\begin{array}{l}\text { parameter in prior for joint distribution of } \\
\text { skill mastery related to easiness/difficulty of } \\
\text { mastery of a skill apriori }\end{array}$ & $\mathbb{R}$ \\
\hline $\mathbf{H}$ & $\begin{array}{l}\text { matrix of edge weights between skills in the } \\
\text { hierarchy which are related to the apriori cor- } \\
\text { relations between skills }\end{array}$ & $\mathbb{R}$ \\
\hline$\tau$ & scaling factor for the edge weights & $\mathbb{R}$ \\
\hline$\omega_{j k}$ & $\begin{array}{l}\text { message value in belief propagation sent from } \\
\text { skill } j \text { to skill } k\end{array}$ & $\mathbb{R}$ \\
\hline$\lambda_{j k}$ & $\begin{array}{l}\text { penalty factor included induce a prerequisite } \\
\text { structure of skill } j \text { for skill } k\end{array}$ & $\mathbb{R}$ \\
\hline
\end{tabular}

Table 2: Notation used in the paper. When not distinguishing between individuals we will drop the index $p$ on $\mathbf{x}_{p}, \mathbf{s}_{p}$, and $\sigma_{p}$. Without loss of generality, and for sake of ease of notation, we will assume that all individuals answer all of the items (and the same items) so $m_{p}=m$ and $\mathbf{i}_{p}=\mathbf{i}=(1, \ldots, m)^{\top}$. 